\title{
4. DATA REPORT: ORGANIC CARBON AND NITROGEN VARIATIONS AT SITE 958, OFF WEST AFRICA ON THE CONTINENTAL SLOPE ${ }^{1}$
}

\author{
Sten Lindblom ${ }^{2}$
}

\section{INTRODUCTION}

Cores taken at Site 958 off the African west coast on the continental slope at a water depth of $3789 \mathrm{~m}$ are primarily nannofossil ooze, but contain distinct olive-green organic-rich turbidites in the upper 80 $\mathrm{m}$ of the 133-m cored sequence (Blum et al., 1996). In this data report, analyses of organic carbon and nitrogen in the sediments at Site 958 are reported, as well as the mineral content of the organic-rich turbidites.

\section{METHODS}

Total carbon and nitrogen were measured on a CHN Leco 900 analyzer. Samples were weighed into tin capsules, which were folded and weighed together with an oxidant. The instrument was calibrated with EDTA (having carbon $=42 \%$ and nitrogen $=9.50 \%$ ). Calibration standards and blanks were run in the beginning and at the end of the samples, with several spaced in between as well. Precision of the instrument is $\pm 0.5 \%$. The overall accuracy of total carbon and nitrogen analyses is $\pm 3 \%$.

The present study focuses on samples collected for carbonate and subsequent analysis of total carbon and nitrogen. Samples were taken from all different types of lithologies, including olive-green intervals with higher organic content. Sample volumes were $5 \mathrm{~cm}^{3}$. The samples were freeze-dried and crushed. Carbonate content (Fig. 1A) was measured on a Coulometrics 5011 carbon dioxide coulometer equipped with a System 140 carbonate carbon analyzer. The overall accuracy of the coulometer measurements is $\pm 3 \%$.

\section{RESULTS \\ Mineral Content}

Sporadic layers of olive-green sediments with fining upward were found down to $88 \mathrm{mbsf}$. The XRD analyses of four olive-green turbidites indicate that they are composed of mainly quartz and calcite (Table 1). The quartz lines may also involve diatoms to some extent. Kaolinite, smectite, and mica are present in the upper two samples, but there is only mica at $22.9 \mathrm{mbsf}$, and only kaolinite and mica at 71 mbsf. A general decrease with depth in mineral content (quartz, biosilica, clay minerals, and volcanic glass) was reported by Blum et al. (1996).

Great fluctuations in mineral content were also indicated down to 45 mbsf. The major lithologic component is nannofossil ooze.

\section{Organic Carbon and Nitrogen}

Organic carbon and nitrogen analyses were made on 32 samples (Table 2). The organic carbon varied between 0 and $2.66 \mathrm{wt} \% \mathrm{C}$ (Fig. 1B). Nitrogen (Fig. 1C) covaries with organic carbon and ranges be-

${ }^{1}$ Firth, J.V. (Ed.), 1998. Proc. ODP, Sci. Results, 159T: College Station, TX (Ocean Drilling Program).

${ }^{2}$ Department of Geology and Geochemistry, Stockholm University, S-106 91, Stockholm, Sweden. Sten_AB.Lindblom@geo-su.se tween 0 and $0.34 \mathrm{wt} \% \mathrm{~N}$. Total organic carbon:nitrogen $(\mathrm{C}: \mathrm{N})$ ratios range between 18.2 and 34.6 (Table 2 and Fig. 1D).

\section{ACKNOWLEDGMENTS}

Anders Sjösten and the Department of System Ecology are thanked for the use of their CHN analyzing equipment. Participation in the scientific description party with John V. Firth, Peter Blum, Klaus Michels, William W. Sager, and Amelie Winkler is acknowledged. XRD analyses were done by Martine Gerard. Ann Pimmel helped set up the coulometer for carbonate analyses.

\section{REFERENCE}

Blum, P., Lindblom, S. Michels, K., Sager, W.W., and Winkler, A., 1996. Site 958. In Firth, J.V., et al., Proc. ODP, Init. Repts., 159T: College Station, TX (Ocean Drilling Program), 3-13.

Date of initial receipt: 16 September 1996

Date of acceptance: 16 May 1997

Ms 159TSR-060

Table 1. XRD data of minerals in the organic-rich turbidites from Hole 958A.

\begin{tabular}{|c|c|c|c|c|}
\hline Sample & $\begin{array}{l}\text { Depth } \\
\text { (mbsf) }\end{array}$ & Main minerals & Accessory minerals & $\begin{array}{c}\text { Organic } \\
\text { carbon } \\
(\mathrm{wt} \%)\end{array}$ \\
\hline \multicolumn{5}{|l|}{ 159T-958A- } \\
\hline $1 \mathrm{H}-3,68 \mathrm{~cm}$ & 3.68 & Quartz, calcite & $\begin{array}{l}\text { Halite, kaolinite, micas, smectites } \\
\text { (rare) }\end{array}$ & 0.71 \\
\hline $3 \mathrm{H}-1,64 \mathrm{~cm}$ & 19.64 & Quartz, calcite & $\begin{array}{l}\text { Halite, kaolinite, micas, smectites } \\
\text { (rare) }\end{array}$ & 1.37 \\
\hline $3 \mathrm{H}-3,94 \mathrm{~cm}$ & 22.94 & Quartz, calcite & Halite, pyrite, micas (altered) & 1.68 \\
\hline $8 \mathrm{H}-4,15 \mathrm{~cm}$ & 71.15 & Quartz, calcite & Halite, kaolinite, micas (altered) & 1.39 \\
\hline
\end{tabular}


Table 2. Carbonate, organic carbon, and nitrogen data from Hole 958A.

\begin{tabular}{crcccc}
\hline Sample & $\begin{array}{c}\text { Depth } \\
(\mathrm{mbsf})\end{array}$ & $\begin{array}{c}\text { Carbonate } \\
(\mathrm{wt} \%)\end{array}$ & $\begin{array}{c}\text { Total organic } \\
\text { carbon }(\mathrm{wt} \%)\end{array}$ & $\begin{array}{c}\text { Total nitrogen } \\
(\mathrm{wt} \%)\end{array}$ & $\begin{array}{c}\text { C:N } \\
\text { ratio }\end{array}$ \\
\hline $159 \mathrm{~T}-958 \mathrm{~A}-$ & & & & & \\
$1 \mathrm{H}-3,68 \mathrm{~cm}$ & 3.68 & 28.07 & 0.711 & 0.037 & 19.2 \\
$1 \mathrm{H}-5,113 \mathrm{~cm}$ & 7.13 & 88.96 & 0.135 & $\mathrm{ND}$ & \\
$2 \mathrm{H}-2,91 \mathrm{~cm}$ & 11.91 & 42.42 & 0.934 & 0.027 & 34.6 \\
$2 \mathrm{H}-4,78 \mathrm{~cm}$ & 14.78 & 45.68 & 0.416 & $\mathrm{ND}$ & \\
$3 \mathrm{H}-1,64 \mathrm{~cm}$ & 19.64 & 33.67 & 1.369 & 0.073 & 23.1 \\
$3 \mathrm{H}-3,94 \mathrm{~cm}$ & 22.94 & 31.66 & 1.677 & 0.092 & 18.2 \\
$3 \mathrm{H}-4,85 \mathrm{~cm}$ & 24.35 & 49.11 & 0.248 & $\mathrm{ND}$ & \\
$4 \mathrm{H}-1,88 \mathrm{~cm}$ & 29.38 & 49.06 & 1.975 & 0.105 & 18.8 \\
$5 \mathrm{H}-2,78 \mathrm{~cm}$ & 40.28 & 74.68 & 0.226 & $\mathrm{ND}$ & \\
$6 \mathrm{H}-2,42 \mathrm{~cm}$ & 49.42 & 51.28 & 0.419 & 0.018 & 23.3 \\
$6 \mathrm{H}-5,102 \mathrm{~cm}$ & 54.52 & 83.08 & 0.208 & $\mathrm{ND}$ & \\
$7 \mathrm{H}-1,80 \mathrm{~cm}$ & 57.80 & 56.53 & 0.139 & $\mathrm{ND}$ & 31.0 \\
$7 \mathrm{H}-5,145 \mathrm{~cm}$ & 64.45 & 54.54 & 1.023 & 0.033 & 19.9 \\
$8 \mathrm{H}-2,119 \mathrm{~cm}$ & 69.19 & 55.40 & 2.661 & 0.134 & \\
$8 \mathrm{H}-4,11 \mathrm{~cm}$ & 71.11 & 61.57 & 0.359 & $\mathrm{ND}$ & 20.4 \\
$8 \mathrm{H}-4,15 \mathrm{~cm}$ & 71.15 & 34.81 & 1.386 & 0.068 & \\
$9 \mathrm{H}-2,76 \mathrm{~cm}$ & 78.26 & 77.32 & 0.036 & $\mathrm{ND}$ & 18.5 \\
$10 \mathrm{H}-2,144 \mathrm{~cm}$ & 88.44 & 37.46 & 1.573 & 0.085 & \\
$14 \mathrm{H}-1,69 \mathrm{~cm}$ & 124.19 & 76.83 & 0.698 & $\mathrm{ND}$ & \\
$159 \mathrm{~T}-958 \mathrm{~B}-$ & & & & & \\
$1 \mathrm{H}-4,30 \mathrm{~cm}$ & 4.80 & 53.41 & 0.064 & $\mathrm{ND}$ & \\
\hline
\end{tabular}

Note: ND = not determined
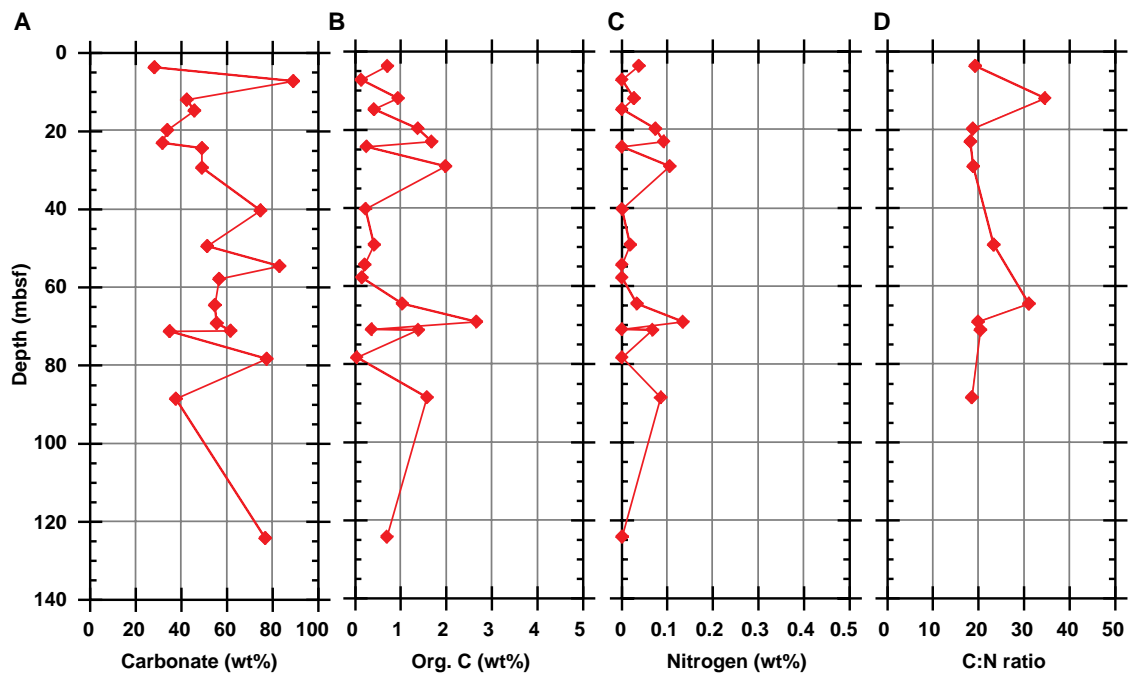

Figure 1. A. Carbonate content vs. depth from Hole 958A (only samples with detectable organic carbon). B. Total organic carbon content vs. depth from Hole 958A. C. Total nitrogen content vs. depth from Hole 958A. D. Total organic carbon/total nitrogen vs. depth from Hole 958A. 